\title{
A WEAK INVARIANCE PRINCIPLE AND ASYMPTOTIC STABILITY FOR EVOLUTION EQUATIONS WITH BOUNDED GENERATORS
}

\author{
By \\ E. N. CHUKWU \\ Department of Mathematics , Box 8205, \\ North Carolina State University \\ Raleigh , N. C. 27695-8205 , U.S.A. \\ and \\ P. SMOCZYNSKI \\ Department of Mathematics and Statistics , \\ Simon Fraser University , \\ Burnaby B. C. , V5A 1 S6, Canada . \\ (Received October 7, 1991 and in revised form April 6, 1993) \\ KEYWORDS : Asymptotic stability, invariance principle , Lyapunov functions \\ AMS SUBJECT CLASSIFICATION CODES : 34C11, 34C20, 36G20 . \\ ABSTRACT. If $\mathrm{V}$ is a Lyapunov function of an equation $\mathrm{du} / \mathrm{dt}=\mathrm{u}^{\prime}=\mathrm{Zu}$ in a Banach space then \\ asymptotic stability of an equilibrium point may be easily proved if it is known that $\sup \left(\mathrm{V}^{\prime}\right)<0$ on \\ sufficiently small spheres centered at the equilibrium point. In this paper weak asymptotic stability is \\ proved for a bounded infinitesimal generator $Z$ under a weaker assumption $V^{\prime} \leq 0$ (which alone \\ implies ordinary stability only) if some observability condition, involving $Z$ and the Frechet derivative \\ of $V^{\prime}$, is satisfied. The proof is based on an extension of LaSalle's invariance principle, which yields \\ convergence in a weak topology and uses a strongly continuous Lyapunov function. The theory is \\ illustrated with an example of an integro-differential equation of interest in the theory of chemical \\ processes . In this case strong asymptotic stability is deduced from the weak one and explicit sufficient \\ conditions for stability are given. In the case of a normal infinitesimal generator $Z$ in a Hilbert \\ space, strong asymptotic stability is proved under the following assumptions : $Z^{*}+Z$ is weakly \\ negative definite and $\operatorname{Ker} Z=\{0\}$. The proof is based on speetral theory .
}


Section 1 . Introduction .

A well-known theorem of A. M. Lyapunov, see [4] or [11] for example, asserts that zero in $R^{n}$ is an asymptotically stable equilibrium point of an evolution equation

$$
\begin{gathered}
\frac{d u}{d t}(t)=Z u(t), t>0 \\
\text { if } V(x)>0 \text { for } x \neq 0, V(0)=0 \text { and } \\
V^{\prime}(x)=(Z x \mid \partial V(x))<0 \text { for } x \neq 0
\end{gathered}
$$

where $V: R^{n} \rightarrow R^{n}$ is a suitable Lyapunov function and $\partial V$ is its Frechet derivative ( notation is further explained in the beginning of Section 2 , asymptotic stability is recalled in Section 3 ). R. Datko in [5] and [6] extended A. M. Lyapunov's theory to infinite-dimensional Banach spaces under a stringent assumption :

$$
\mathbf{V}^{\prime}(\mathrm{x})=(\mathrm{Zx} \mid \partial \mathrm{V}(\mathrm{x})) \leq-\alpha(\|\mathrm{x}\|)
$$

where $\alpha:[0,+\infty) \rightarrow[0,+\infty), \alpha(x)>0$ for $x>0$ and $\alpha(0)=0$, ( see also remarks and papers quoted in [18]). If $V(x)=(x \mid G x)$, where $G$ is a symmetric operator, then the above inequality means strict negative definiteness of the operator $Z^{*} G+G Z$, where $Z^{*}$ is the adjoint of $Z$. One is compelled to use assumptions as this by the fact that bounded sets in a Banach space are precompact if and only if the space is finite-dimensional, see [ 7]. However, if one manages to find a Lyapunov function that satisfies a weaker condition

$$
\mathbf{V}^{\prime}(\mathbf{x})=(\mathrm{Zx} \mid \partial \mathbf{V}(\mathbf{x}))>0 \text { for } \mathbf{x} \neq 0,
$$

then Lyapunov's theory yields only ordinary stability even in the finite-dimensional case [ 4].

Essential information on asymptotic behavior of solutions under (1.4) is given in LaSalle's invariance principle, see [ 13], according to which all the solutions converge to the maximal invariant subset $M$ of $R=\left\{x: V^{\prime}(x)=0\right\}$. Today this principle is a standard tool of investigation of asymptotic stability in Banach spaces, see [ 12], [ 11], [ 18] (the context of wave equation) and [9], [ 2], [ 3] (the context of functional differential equations).

Using LaSalle's invariance principle J.P. Miller and A. N. Mitchell, see [ 14], proved in 1980 an extremely interesting fact : stability for a linear system in a finite dimensional case under (1.4) is equivalent to observability of the pair $\left(\mathrm{Z}, \partial \mathrm{V}^{\prime}\right)$, ( observability is explained at the beginning of Section 3 ). This idea proved fruitful in theory of retarded differential equations, see [ 2 ], [ 3 ] .

Strong compactness of trajectories is characteristic for wave equations, functional differential equations and parabolic equations. This is related with the fact that the generators are unbounded and in this aspect bounded generators, with weak damping, are not easier to treat than unbounded ones : if compactness of the resolvent is lost then only weak asymptotic stability may be obtained. However, strong asymptotic stability may be easily deduced from the weak one if strong compactness of trajectories may be proved independently of compactness of the resolvent .

Evolution equations (1.1) in Banach spaces with a linear bounded infinitesimal generators $\mathbf{Z}$ are considered in this paper. Weak asymptotic stability is proved with the help of a Lyapunov function with non-strictly negative time derivative. Section 2 deals with omega-limit sets. It is shown that the LaSalle's maximal invariant set coincides with the unobservable space of the pair $\left(Z, \partial V^{\prime}\right)$ and the weak invariance principle is proved. Weak asymptotic stability is proved in Section 3 if, moreover, the pair $\left(Z, \partial V^{\prime}\right)$ is observable. By means of a simple example it is shown that observability is not a necessary condition of weak asymptotic stability. The theory is applied to equations with Volterra integral operators in the right-hand sides that arise in the theory of chemical processes, see [1]. For this type of operator the kernel of $\partial V^{\prime}$ may be of codimension one and observability is unexpected. Simple conditions on the kernel of the integral operator that ensure strong stability are stated . Normal operators in Hilbert spaces are considered in Section 4 without the technique of invariant sets and compactness arguments. It is also shown with the help of an example that under weak damping considered in this paper trajectories tend to equilibrium much more slowly than in the case of a strong 
damping corresponding to (1.3) . R. Datko proved that for strictly negative definite generators in Hilbert spaces trajectories tend exponentially to zero. It is proved in [21] that exponential weak asymptotic stability implies exponential strong stability (the adjective exponential is omitted in [21]).

Section 2 . Weak invariance principle and non-observability .

We consider a linear evolution equation

$$
\frac{\mathrm{du}}{\mathrm{dt}}(\mathrm{t})=\mathrm{Zu}(\mathrm{t}), \mathrm{t}>0,
$$

where $\mathrm{Z}: \mathrm{B} \rightarrow \mathrm{B}$ is a bounded linear operator in a Banach space B. See [ 16] for existence and uniqueness of solutions and for the proof that $u(t)=e^{Z\left(t-t_{0}\right)}\left(u\left(t_{0}\right)\right)$ for every solution $u$ and all $t_{1} t_{1} \geq 0$.

We shall use frequently the following notation. $B$ is a Banach space over the field $C$ of complex numbers, $B^{*}$ its adjoint space and $(.1):. B \times B^{*} \rightarrow C$ the duality mapping. If $x \in B$ then its norm is denoted as $\|x\|$. We assume that $\left(z_{1} x \mid z_{2} y\right)=z_{1} \bar{z}_{2}(x \mid y)$ for all $z_{1}, z_{2} \in C$ and all $x \in B, y \in B^{*}$, where the bar denotes conjugation. A sequence $\left\{z_{n}, n=1,2, \ldots\right\} \mid B$ converges weakly to $z_{\infty} \in B$ if $\lim _{n \rightarrow \infty}\left(z_{n}-z_{\infty} \mid y\right)=0$ for all $y \in B^{*}$ and this will be written shortly as $\mathrm{z}_{\infty}=\mathrm{w}-\lim _{\mathrm{n} \rightarrow \infty} \mathrm{z}_{\mathrm{n}}$.

Definition 2.1. Let $u:[0,+\infty) \rightarrow B$. Then $x \in B$ belongs to the weak omega-limit set of $u$, shortly $\mathrm{x} \in \mathrm{w \omega ls}(\mathrm{u})$ if

$$
\liminf _{t \rightarrow \infty}|(u(t)-x \mid y)|=0 \text { for all } y \in B^{*}
$$

and belongs to the strong omega-limit set of $\mathrm{u}$, shortly $\mathrm{x} \in \operatorname{s\omega ls}(\mathrm{u})$ if $\liminf _{\mathrm{t} \rightarrow \infty}\|\mathrm{u}(\mathrm{t})-\mathrm{x}\|=0$.

We need two lemmas in the proof of the invariance principle .

Lemma 2.2 . ON OMEGA-LIMTT SETS . Let $\mathrm{u} \in \mathrm{C}^{1}((0,+\infty)$, B ) be a bounded solution of an evolution equation (2.1) with a bounded infinitesimal generator $\mathrm{Z}$ in a reflexive Banach space $\mathrm{B}$. Then $\omega \omega \mathrm{ls}(\mathrm{u})$ is a non-empty weakly compact invariant subset of $\mathrm{B}, \mathrm{s} \omega \mathrm{ls}(\mathrm{u})$ is a non-empty strongly compact invariant subset of $\mathrm{B}$ and $\operatorname{s\omega ls}(\mathrm{u}) \subseteq \mathrm{w} \omega \mathrm{ls}(\mathrm{u})$.

The proof of this lemma is omitted since it is similar to the proofs of the corresponding statements in [11] and [ 18]. The second lemma is quite elementary and its proof is omitted, but it supports an essential step of the proof of the invariance principle.

Lemma 2.3. If $\mathrm{f} \in \mathrm{C}^{\mathrm{l}}((0,+\infty),[0,+\infty)), \mathrm{f}^{\prime}=\mathrm{df} / \mathrm{dt} \leq 0$ in $[0,+\infty)$ and $\mathrm{f}^{\prime}$ is uniformly continuous in $(0,+\infty)$ then $\lim _{\mathrm{t} \rightarrow \infty} \mathrm{f}^{\prime}(\mathrm{t})=0$.

In order to formulate precisely the invariance principle we recall the following notions . A bounded linear operator $A: B \rightarrow B^{*}$ in a Banach space $B$ is called symmetric if $(x \mid A y)=(y \mid A x)^{-}$for all $x, y \in B$, where the bar denotes conjugation, hence $(x \mid A x)$ is real . A symmetric bounded linear operator $A$ is called weakly negative (positive) definite if $(y ! 4 y) \leq(\geq) 0$ for all $y \in B$, and is called strictly positive if $(y \mid A y) \geq\|y\|^{2}$ for all $y \in B$.

Let $G: B \rightarrow B^{*}$ be a symmetric bounded linear operator. Let $u \in C^{1}((0,+\infty), B)$ be a solution of $(2.1)$ and let $V(x)=(x \mid G x)$. Then the time derivative of $V(u(t))$ may be expressed as follows :

$$
V^{\prime}(u(t))=\frac{d V(u)}{d t}(t)=\left(u(t) \mid\left(Z^{*} G+G Z\right) u(t)\right)
$$

where $Z^{*}: B^{*} \rightarrow B^{*}$ is the adjoint of $Z . V(x) \rightarrow R^{1}$ for all $x$ in $B$ by symmetry of $G$, similarly $V^{\prime}(x) \in R^{1}$, hence $V: B \rightarrow R^{1}$ may serve as a Lyapunov function .

The major advantage of the result below over Theorem 2.3 in $[18]$ is that the Lyapunov function may be only strongly continuous and needs not be weakly continuous .

Theorem 2.4. WEAK INVARIANCE PRINCIPLE. Let $\mathrm{u} \in \mathrm{C}^{1}((0,+\infty)$, B $)$ be a solution of (2.1) with a bounded infinitesimal generator $\mathrm{Z}$ in a reflexive Banach space $\mathrm{B}$. Let $\mathrm{G}: \mathrm{B} \rightarrow \mathrm{B}^{*}$ be a symmetric bounded strictly positive linear operator such that the operator $\mathrm{Z}^{*} \mathrm{G}+\mathrm{GZ}: \mathrm{B} \rightarrow \mathrm{B}^{*}$ is 
weakly negative definite. Let $\mathrm{M}$ be the maximal invartant set of (2.1) contained in $\operatorname{Ker}\left(Z^{*} \mathrm{G}+\mathrm{GZ}\right)$. Then $\mathrm{u}(\mathrm{t})$ approaches $\mathrm{M}$ in the following sense:

$$
\inf \left\{\liminf _{\mathrm{t} \rightarrow \infty}|(\mathrm{u}(\mathrm{t})-\mathrm{x} \mid \mathrm{y})|: \mathrm{x} \in \mathrm{M}\right\}=0 \text { for all } \mathrm{y} \in \mathrm{B}^{*}
$$

Proof . Let us take $V(x)=(x \mid G x)$ as a Lyapunov function. Then $\|\mathrm{u}(\mathrm{t})\|^{2} \leq \mathrm{V}(\mathrm{u}(\mathrm{t})) \leq \mathrm{V}(\mathrm{u}(0)) \leq+\infty$ since $\mathrm{V}^{\prime}(\mathrm{u}(\mathrm{t})) \leq 0$ by $(2.5)$ and weak negative definiteness of $Z^{*} G+G Z$, so $u(t)$ is bounded. All the other assumptions of Lemma 2.2 are also satisfied, hence $w \omega l s(u)$ is a non-empty and weakly compact invariant set. If $w \omega l s(u) \subseteq \operatorname{Ker}\left(Z^{*} G+G Z\right)$ then (2.6) follows from (2.3) since $w \omega l s(u) \subseteq M$ by the definition of $M$ and invariance of $w \omega l s(u)$. Therefore the proof will be complete if we show that $w \omega l s(u) \subseteq \operatorname{Ker}(Z * G+G Z)$.

So, let $x=w-\lim _{n \rightarrow \infty} u\left(t_{n}\right) \in w \omega l s(u)$. Using (2.5) we obtain the following identity :

$$
\begin{aligned}
V^{\prime}\left(u\left(t_{n}\right)-x\right)= & \left(u\left(t_{n}\right) \mid\left(Z^{*} G+G Z\right) u\left(t_{n}\right)\right)+\left(x \mid\left(Z^{*} G+G Z\right) x\right) \\
& -\left(u\left(t_{n}\right) \mid\left(Z^{*} G+G Z\right) x\right)-\left(x \mid\left(Z^{*} G+G Z\right) u\left(t_{n}\right)\right) \\
= & V^{\prime}\left(u\left(t_{n}\right)\right)+V^{\prime}(x)-2 \operatorname{Re}\left(u\left(t_{n}\right) \mid\left(Z^{*} G+G Z\right) x\right) .
\end{aligned}
$$

The left-hand side is non-positive by the weak negative definiteness of $Z * G+G Z$. The first term in the right-hand side converges to zero by Lemma (2.3), for $V^{\prime}(u(t))$ is a non-positive and uniformly continuous function of $t$, thanks to boundedness of $G$ and $Z$. The third term converges to $2 \operatorname{Re}\left(x \mid\left(Z^{*} G+G Z\right) x\right)=-2 V^{\prime}(x)$, where Re denotes the real part. Thus taking limsup of both sides we obtain that $0 \geq V^{\prime}(x)-2 V^{\prime}(x)=-V^{\prime}(x)$ hence $V^{\prime}(x) \geq 0$. This, together with $V^{\prime}(x) \leq 0$, which follows from (2.5) and the weak negative definiteness of $Z^{*} G+G Z$, implies $V^{\prime}(x)=0$.

Let us consider the following function of a real variable $s$ :

$$
g(s)=V^{\prime}(x+y s)=\left(x+y s \mid\left(Z^{*} G+G Z\right)(x+y s)\right),
$$

where $x, y \in B$ are fixed. Since $V^{\prime}$ takes only non-positive values by the weak negative definiteness of $Z^{*} G+G Z$, then $g \leq 0$. It was shown above that $g(0)=V^{\prime}(x)=0$, thus $g$ attains its maximum value for $s=0$, hence $2 \operatorname{Re}\left(y \mid\left(Z^{*} G+G Z\right) x\right)=g^{\prime}(0)=0$. Replacing $y$ with $\sqrt{ }(-1) y$ we obtain in a similar way $2 \operatorname{Im}\left(y \mid\left(Z^{*} G+G Z\right) x\right)=0$, therefore $(y \mid(Z * G+G Z) x)=0$, which implies that $(Z * G+G Z) x=0$, for $y$ may be any element of $B$. Thus we have showed that w wls $(u)$ is a subset of $\operatorname{Ker}\left(Z^{*} G+G Z\right)$. Q.E.D.

Remark. If $B$ is separable then formulas (2.6) and (2.3) may be given a metric form as in [ 18, Corollary 2.1].

The maximal invariant set from LaSalle's invariance principle may be described precisely using the notion of non-observability from control theory. Let $\mathrm{W}: \mathrm{B} \rightarrow \mathrm{B}^{*}$ and $\mathrm{Z}: \mathrm{B} \rightarrow \mathrm{B}$ be two bounded linear operators. Then the closed subspace of B

$$
\operatorname{Nobs}(Z, W)=\bigcap_{n \geq 0} \operatorname{Ker} W Z^{n}
$$

is the non-observable space of the pair $(\mathrm{Z}, \mathrm{W})$. (Compare this definition with the sufficient condition of observability (3.1) in Section 3 ). It seems that the following simple fact remained unrecorded till this time .

Lemma 2.5. ON MAXIMAL INVARIANT SET. If $\mathrm{Z}$ and $\mathrm{W}$ are bounded, then $\operatorname{Nobs}(Z, W)$ coincides with the maximal invariant subset of (2.1) that is contained in Ker W.

Proof . In the first part we prove that $\operatorname{Nobs}(Z, W)$ is an invariant subset of $\operatorname{Ker} W$. By its definition $\operatorname{Nobs}(Z, W)$ is a subspace of $W$, hence it remains to show invariance , that is ,

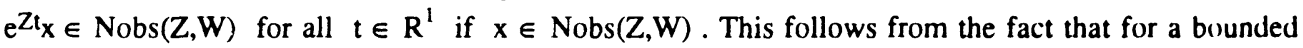


operator $Z, e^{Z t}=\lim _{K \rightarrow \infty} \sum_{k=0}^{K}(Z t)^{k} / k$ ! in the sense of the uniform operator topology, see $[17]$, hence by (2.7): $\quad W Z^{n} e^{Z t_{x}}=\lim _{K \rightarrow \infty} \sum_{k=13}^{K}(Z t)^{k} / k !=0$.

Second part : maxımality . Let $M$ denote the maximal invariant subset of Ker W . Maximality of $M$ implies that $\operatorname{Nobs}(Z, W) \subseteq M$. It remains to show the converse inclusion. Let us consuder an arbitrary element $x$ of $M$. Then $W^{Z t} x=0$ for $M$ is invariant and $M \subseteq K e r W$. The function $\mathrm{We}^{\mathrm{Z} \mathrm{t}_{\mathrm{x}}}$ is infinitely many times differentiable since $\mathrm{Z}$ and $\mathrm{W}$ are bounded, hence all its derivatives are equal zero for $t \geq 0$, that is $W Z^{n} e^{Z} t x=0$ for all $n \geq 0$ and $t \geq 0$. Taking $t=0$ we conclucle that $x \in \operatorname{Nobs}(Z, W)$, thus $M \subseteq \operatorname{Nobs}(Z, W)$, for $x$ was arbitrary. Therefore $M=\operatorname{Nobs}(Z, W)$.

Corollary 2.6. ON LASALLE'S MAXIMAL INVARIANT SET. Under the assumptions of Theorem 2.4 the maximal invariant set $M$ coincides with $\operatorname{Nobs}(Z, Z * G+G Z)$.

Proof. This follows from Theorem 2.5 with $W=Z^{*} G+G Z$.

Section 3 . Weak asymptotic stability and observability .

Weak asymptotic stability for the evolution equation (2.1) is proved in this sectuon with the help of observability. The pair ( $\mathrm{Z}, \mathrm{W})$ is called observable if for every solution $\mathrm{u}$ of (2.1) the condition $W u(t)=0$ for all $t>0$ implies that $u(t)=0$ for all $t>0$. A necessary and sufficient condition for observability for bounded operators is that ( see [ 19, first part of Theorem 5.1.1]) :

$$
\operatorname{Nobs}(Z, W)=\bigcap_{n \geq 0} \operatorname{Ker} W Z^{n}=\{0\} .
$$

In order to make communication precise, let us maintain the following well-known terminology . Zero in B , the equilibrium point of $(2.1)$, is :

a) stable if for every $\varepsilon>0$ there exists a $\delta>0$ such that for every solution u we

have that $\|\mathrm{u}(\mathrm{t})\| \leq \varepsilon$ for all $\mathrm{t} \geq 0$, whenever $\|\mathrm{u}(0)\| \leq \delta$. This is called also ordinary stability .

b) asymptotically stable if $\lim _{\mathrm{t} \rightarrow \infty}\|\mathrm{u}(\mathrm{t})\|=0$ for every solution $\mathrm{u}$.

This is called also strong asymptotic stability .

c) weakly asymptotically stable if $\mathrm{w}-\lim _{\mathrm{t} \rightarrow \infty} \mathrm{u}(\mathrm{t})=0$ for every solution $\mathrm{u}$.

The principal result of this paper is as follows :

Theorem 3.1 . ON WEAK ASYMPTOTIC STABILITY . Let $\mathrm{u} \in \mathrm{C}^{1}((0,+\infty), \mathrm{B})$ be a solution of (2.1) with a bounded infinitesimal generator $\mathrm{Z}$ in a reflexive Banach space $\mathrm{B}$. Let $\mathrm{G}: \mathrm{B} \rightarrow \mathrm{B}^{*}$ be a symmetric bounded strictly positive linear operator such that the operator $\mathrm{Z}^{*} \mathrm{G}+\mathrm{GZ}: \mathrm{B} \rightarrow \mathrm{B}^{*}$ is weakly negative definite and the pair $(\mathrm{Z}, \mathrm{Z} * \mathrm{G}+\mathrm{GZ})$ is observable. Then zero in B is a weakly asymptotically stable equilibrium point of (2.1).

Proof. All the assumptions of Theorem 2.1 are satisfied, hence $u(t)$ converges in the sense of formula (2.6) to the maximal invariant subset $M$ of $\operatorname{Ker}\left(Z^{*} G+G Z\right)$. Corollary 2.6 implies that $M=\operatorname{Nobs}(Z, Z * G+G Z)$, hence $M=\{0\}$ by the observability assumption. Therefore, by (2.6), we have that for every solution $u$ of $(2.1) \liminf _{t \rightarrow \infty}|(u(t) \mid y)|=0$ for all $y \in B^{*}$. Suppose that for some $\mathrm{y}_{1} \in \mathrm{B}^{*}$

$$
\limsup _{t \rightarrow \infty}\left|\left(u(t) \mid y_{1}\right)\right|=r>0 \text {. }
$$

Then there exists a sequence $\left\{t_{n}, n=1,2, \ldots ..\right\}$ as in Definition (2.1) such that $\lim _{n \rightarrow \infty}\left(u\left(t_{n}\right) \mid y_{1}\right)$ exists and is equal to $r$. Now boundedness of $u$, reflexivity of $B$ and the Eberlain-Shmulyan theorem imply that there exists a subsequence $\left\{t_{n_{j}}, j=1,2, \ldots\right\}$ such that $u_{\infty}=w-\lim _{j \rightarrow \infty} u\left(t_{n_{j}}\right)$ exists and then $\left|\left(u_{\infty} \mid y_{1}\right)\right|=r \neq 0$ by (3.2), hence $u_{\infty} \neq 0$. But $u_{\infty}$ is an element of wwls(u) which is a subset of $M$. Thus a non-zero $u_{\infty}$ belongs to $M$, which, by observability, consists of zero alone - a contradiction. Therefore $w-\lim _{t \rightarrow \infty} u(t)=0$. Q.E.D.

Remark 3.2 . J. P. Miller and A. N. Mitchell proved in [14] that , under (1.4), observability is a necessary and sufficient condition of asymptotic stability. Theorem 3.1 asserts only sufficiency of 
this condition for weak asymptotic stability. It follows from the Example below that observabulity is not a necessary condition of weak asymptotic stability. Theorem 3.3 shows however that strong asymptotic stability implıes observability .

Example. Let $B^{*}=B=L^{2}([0,1], C)$ and let $Z x(s)=\sqrt{ }(-1) \cdot s \cdot x(s)$,

$\mathrm{G}(\mathrm{x}, \mathrm{y})=\int_{[0.1]} \mathrm{x}(\sigma) \mathrm{y}(\sigma) \mathrm{d} \sigma$. Let us consider an equation $\partial_{\mathrm{t}} \mathrm{u}(\mathrm{t}, \mathrm{s})=\mathrm{Zu}(\mathrm{s})=\sqrt{ }(-1) \cdot \mathrm{s} \cdot \mathrm{x}(\mathrm{s})$. Then

$$
\mathrm{V}(\mathrm{x})=\mathrm{G}(\mathrm{x}, \mathrm{x})=(\mathrm{x} \mid \mathrm{x})=\|\mathrm{x}\|^{2}=\int_{[0,1]}|\mathrm{x}(\sigma)|^{2} \mathrm{~d} \sigma
$$

may serve as a Lyapunov function. Next $Z^{*} G+G Z=0$, hence $V^{\prime}(u(t))=0$ by (2.5). Therefore all the solutions are bounded. The solution of the above equation is given by the following formula : $u(t, s)=u(0, s) e^{s t} \sqrt{ }(-1)$, therefore

$$
\lim _{t \rightarrow \infty} \int_{[0,1]} u(t, s) \cdot v(s) d s=\lim _{t \rightarrow \infty} \int_{[0,1]} u(0, s) e(\sqrt{ }(-1) s t) \cdot v(s) d s=0 \text { for every } v \in B,
$$

hence $w-\lim _{t \rightarrow \infty} u(t,)=$.0 , thus zero is an asymptotically stable equilibrium point. However the observability condition (3.1) is not satisfied for in this case :

$\operatorname{Nobs}\left(Z, Z^{*}+Z\right)=\bigcap_{n \geq 0} \operatorname{Ker}\left(Z^{*}+Z\right) Z^{n}=\operatorname{Ker}\left(Z^{*}+Z\right)=H \neq(0)$.

Theorem 3.3. ON OBSERVABILITY FROM ASYMPTOTIC STABILITY . Let G:B $\rightarrow$ B* be a symmetric bounded strictly positive linear operator. If $\mathrm{Z}$ is a bounded operator on a Banach space $\mathrm{B}$ such that the operator $\mathrm{Z}^{*} \mathrm{G}+\mathrm{GZ}: \mathrm{B} \rightarrow \mathrm{B}^{*}$ is weakly negative definite and zero in $\mathrm{H}$ is a strongly asymptotically stable equilibrium point of (2.1) then the pair $\left(\mathrm{Z}, \mathrm{GZ}+\mathrm{Z}^{*} \mathrm{G}\right)$ is observable.

Proof . Let $0 \neq x \in \operatorname{Nobs}(Z, G Z+Z * G)$. Then $u(t)=e^{Z t} x \in \operatorname{Nobs}(Z, G Z+Z * G)$ for $\operatorname{Nobs}\left(Z, G Z+Z^{*} G\right) \quad$ is an invariant subspace of (2.1) by Theorem 2.5 , hence $\frac{\mathrm{d}(\mathrm{Gulu})}{\mathrm{dt}}=(\mathrm{u} \mid(\mathrm{GZ}+\mathrm{Z} * \mathrm{G}) \mathrm{u})=0$ since $\operatorname{Nobs}\left(\mathrm{Z}, \mathrm{GZ}+\mathrm{Z}^{*} \mathrm{G}\right) \subseteq \operatorname{Ker}(\mathrm{GZ}+\mathrm{Z} * \mathrm{G})$, hence $(\mathrm{Gu}(\mathrm{t}) l \mathrm{u}(\mathrm{t}))=(\mathrm{Gu}(0) \mathrm{u}(0))$. Thus $\mathrm{u}(\mathrm{t})$ does not tend strongly to zero. This contradicts the assumption of strong asymptotic stability . Q.E.D.

\section{Section 4 . Strong stability for integral generators .}

If we take a Hilbert space $\mathrm{H}$ as the Banach space $\mathrm{B}$ in Theorems 2.4 and 3.1 then $B^{*}=H$ by the Riesz representation theorem and the duality mapping coincides with the inner product. We take $G$ to be the identity operator so that $V(x)=(x \mid x)=\|x\|^{2}$. Theorem 3.1 is now applicable if the operator $\mathrm{W}=\mathrm{Z}^{*} \mathrm{G}+\mathrm{GZ}=\mathrm{Z}^{*}+\mathrm{Z}$ is weakly negative definite and the pair $(\mathrm{Z}, \mathrm{W})$ satisfies the observability condition (3.1). We apply this theory to simple operators. A subspace $S$ of a Hilbert space is an invariant subspace of a bounded linear operator $K: H \rightarrow H$ if $K S \subseteq S$. A bounded linear operator $\mathrm{K}: \mathrm{H} \rightarrow \mathrm{H}$ is called simple if $\mathrm{K}$ and $\mathrm{K}^{*}$ have no common invariant subspace on which they coincide. $\mathrm{K}$ is simple if and only if the following controllability condition holds :

closure $\left\{\bigcup_{\mathrm{n} z 0} \operatorname{Ran} K^{\mathrm{n}}\left(\mathrm{K}-\mathrm{K}^{*}\right)\right\}=\mathrm{H}$, see $[8$, chapter 1 , section 7 , point 1 , property 3$]$. This, by duality (see [ 19 ], the proof of Theorem 5.1.1), is equivalent to an observability condition :

$$
\bigcap_{n \geq 0} \operatorname{Ker}\left(K-K^{*}\right) K^{*^{n}}=\{0\},
$$

The following special case of Theorem 3.1 is of some interest in the theory of integrodifferential equations .

Theorem 4.1 . ON ASYMPTOTIC STABILITY FOR SIMPLE GENERATORS . If the infinitesimal generator $\mathrm{Z}$ of the evolution equation $(2.1)$ is a bounded weakly negative definite operator in a Hilbert space such that $\mathrm{K}=\sqrt{ }(-1) \mathrm{Z}^{*}$ is a simple operator, then zero in $\mathrm{H}$ is a weakly asymptotically stable equilibrium point of (2.1). 
Proof . $K=v(-1) Z^{*}$ is simple hence (4.3) implies the following observability condition : $\bigcap_{n \geq 0} \operatorname{Ker}\left(Z+Z^{*}\right) Z^{n}=\{0\}$, thus the pair $\left(Z, Z+Z^{*}\right)$ is observable. All the assumptuons of Theorem 3.1 are satisfied if one takes the identity operator as the operator $G$, the result follows . Q.E.D.

In the proof of the next theorem we shall need a theorem on approximations by compact sets. If $X, Y$ are two bounded strongly closed subsets of a Banach space $B$ then their

Hausdorff distance $\rho(X, Y)=\sup _{x \in X} \inf _{y \in Y}\|x-y\|+\sup _{y \in Y} \inf _{x \in X}\|x-y\|$ is well defined and it induces a metric topology in the family of bounded closed subsets of $B$.

Lemma 4.2 . ON COMPACT SETS. The family of compact sets is a closed subset of the topological space of closed sets equipped with Hausdorff metrics .

The idea of proof. The definition : "a subset of a metric space is strongly compact if for every positive $\varepsilon$ there exists such a finite set of balls of radius $\varepsilon$ that covers it" means that the centers of the balls approximate the set in Hausdorff metric, therefore the family of compact sets is the closure under Hausdorff metric of the family of all the finite sets, hence a closed set .

Theorem 4.3 . ON ASYMPTOTIC STABILITY FOR INTEGRAL GENERATORS . Let $z(x, s)$ be a measurable function defined for all $\mathrm{s}, \mathrm{x} \in[0,1]$ such that $\mathrm{z}(\mathrm{x}, \mathrm{s})=0$ for $\mathrm{s} \geq \mathrm{x}$ and $\mathrm{z}(\mathrm{x}, \mathrm{x}) \neq 0$ for all $\mathrm{x} \in[0,1]$ and let the integral operator in $\mathrm{L}^{2}([0,1], \mathrm{C})$ given by $\mathrm{Zu}(\mathrm{x})=\int_{[0, \mathrm{x}]} \mathrm{z}(\mathrm{x}, \mathrm{s}) \mathrm{u}(\mathrm{s}) \mathrm{ds}$ be weakly negative definite. Then zero in $\mathrm{L}^{2}([0,1], \mathrm{C})$ is a weakly asymptotically stable equilibrium point of

$$
\partial_{\mathrm{t}} \mathrm{u}(\mathrm{t}, \mathrm{s})=\int_{[0, \mathrm{x}]} \mathrm{z}(\mathrm{x}, \mathrm{s}) \mathrm{u}(\mathrm{s}) \mathrm{d} \mathrm{s} .
$$

If , moreover, $\mathrm{z}(\mathrm{x}, \mathrm{s})$ is continuous with respect to $\mathrm{s}, \partial_{\mathrm{x}} \mathrm{z}(\mathrm{x}, \mathrm{s})$ is a measurable function (for $\left.\mathrm{s} \leq \mathrm{x}\right)$ and the operator

$$
(\mathrm{u}, \mathrm{v}) \rightarrow\left(\int_{[0, \mathrm{x}]} \mathrm{z}(\mathrm{x}, \mathrm{s}) \mathrm{u}(\mathrm{s}) \mathrm{ds}, \int_{[0, \mathrm{x}]} \partial_{\mathrm{x}} \mathrm{z}(\mathrm{x}, \mathrm{s}) \mathrm{u}(\mathrm{s}) \mathrm{d} \mathrm{s}+\mathrm{z}(\mathrm{x}, \mathrm{x}) \int_{[0, \mathrm{x}]} \mathrm{v}(\mathrm{s}) \mathrm{ds}\right)
$$

is also weakly negative definite, then zero is strongly asymptotically stable .

Proof . Since $\mathrm{Z}$ is weakly negative definite, then weak stability follows from Theorem 4.1 if we prove that $K=\sqrt{ }(-1) Z^{*}$ is a simple operator. From Theorem 7.1 , section 7 of $[8]$ it follows that

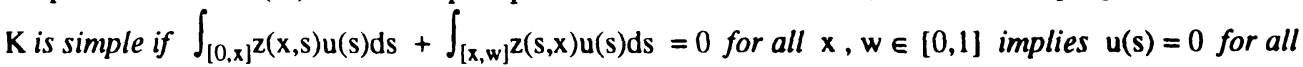
$s \in[0,1]$. Taking $w=x$ we obtain that the first term has to be zero, hence the second has to be also zero. The continuity assumption and the requirement $z(x, x) \neq 0$ imply that $u(s)=0$ for all $s \in[0,1]$, hence $\sqrt{ }(-1) Z^{*}$ is simple and weak asymptotic stability is proved

Now let us consider (4.4) in another Hilbert space :

$$
H_{1}=\left\{u \in L^{2}([0,1], C): \partial u \in L^{2}([0,1], C), u(0)=0\right\} \text {. }
$$

The equation (4.4) in this space is equivalent to a system of two equations in $L^{2}([0,1], C) \oplus L^{2}([0,1], C)$ consisting of (4.4) and an equation for the derivative :

$$
\partial_{t} \partial_{x} u(t, s)=\int_{[0, x]} \partial_{x} z(x, s) u(t, \sigma) d \sigma+z(x, x) \int_{[0, x]} \partial_{x} u(t, \sigma) d \sigma \text {. }
$$

Therefore zero in $\mathrm{L}^{2}([0,1], C) \oplus \mathrm{L}^{2}([0,1], \mathrm{C})$ is a weakly asymptotically stable equilibrium point of the system by the already proved part on weak stability, hence also in $\mathrm{H}_{1}$. Trajectories are bounded in $\mathrm{H}_{1}$, hence by Sobolev imbedding theorem these are precompact in the strong topology of $L^{2}([0,1], C)$, so they tend to their strong omega-limit sets in the sense of strong topology of $\mathrm{L}^{2}([0,1], \mathrm{C})$. But the strong omega-limit set is contained in the weak omega-limit set that consist of zero alone. Thus strong asymptotic stability is proved provided the trajectory starts from $H_{1}$. Now let $x \in L^{2}([0,1], C)$. Since $H_{1}$ is dense in $L^{2}([0,1], C)$, then there exists a sequence $x_{j} \in H_{1}$ for $j=1,2, \ldots$ which strongly converges in $L^{2}([0,1], C)$ to $x$ and therefore $e^{Z} t_{x_{j}}$ converges strongly to $e^{Z} t_{x}$ for $\left\|e^{Z t}\right\|$ is bounded independently of $t$. Thus the sequence of trajectories converges under Hausdorff metric in the topological space of closed subsets of $L^{2}([0,1], C)$ to the trajectory starting 
from $x$. Each trajectory starting from an $x_{j}$ is strongly precompact and, hence also the trajectory starting from $x$ is strongly precompact by Lemma 4.2 . Q.E.D.

Proposition 4.4. ON NEGATIVENESS. The operator from (4.4) is weakly negative definite if for all finite non-decreasing sequences $x_{j}, j=1,2,3, \ldots, N+N_{1} \operatorname{det}\left(M+M^{*}\right) \geq 0$, where $M_{1, j}=z\left(x_{1}, x_{j}\right)$ for $1 \leq \mathrm{j} \leq \mathrm{i} \leq \mathrm{N}$ and $\mathrm{M}_{1, \mathrm{j}}=0$ for $1 \leq \mathrm{i}<\mathrm{j} \leq \mathrm{N}$. Similarly, the operator from (4.5) is weakly negative definite if $\operatorname{det}\left(\mathrm{L}_{+} \mathrm{L}^{*}\right) \geq 0$ where $\mathrm{L}_{1, \mathrm{j}}=\mathrm{M}_{\mathrm{i}, \mathrm{j}}$ for $1 \leq \mathrm{i}, \mathrm{j} \leq \mathrm{N}, \mathrm{L}_{\mathrm{i}, \mathrm{j}}=\partial_{\mathrm{x}} \mathrm{z}\left(\mathrm{x}_{1}, \mathrm{x}_{\mathrm{j}}\right)$ for $\mathrm{N}<\mathrm{N}+\mathrm{j} \leq \mathrm{i} \leq \mathrm{N}+\mathrm{N}_{1}$ and $\mathrm{L}_{1, \mathrm{j}}=0$ for $\mathrm{N}<\mathrm{i}<\mathrm{N}+\mathrm{j} \leq \mathrm{N}+\mathrm{N}_{1}, \mathrm{~L}_{1, \mathrm{j}}=0$ for $1 \leq \mathrm{i} \leq \mathrm{N}$ and $\mathrm{N}<\mathrm{j} \leq \mathrm{N}+\mathrm{N}_{1}, \mathrm{~L}_{1, j}=z\left(\mathrm{x}_{1}, \mathrm{x}_{1}\right)$ for $\mathrm{N}<\mathrm{j} \leq \mathrm{i} \leq \mathrm{N}+\mathrm{N}_{1}$ and $\mathrm{L}_{1, \mathrm{j}}=0$ for $\mathrm{N}<\mathrm{i}<\mathrm{N}+\mathrm{j} \leq \mathrm{N}+\mathrm{N}_{1}$.

The conditions for negativeness of the operator from (4.5) are satisfied if the operator from (4.4) is negative-definite and the kernel $z(x, s)$ is independent of the first argument .

\section{Section 5. Strong asymptotic stability for normal generators .}

Equivalence of asymptotic stability and observability for equations (2.1) with weakly dissipative normal infinitesimal generators, Theorem 5.3 , is proved in this section. The proof is decomposed into three parts, two of these are interesting in themselves and are stated as separate theorems. The first one of these is independent of normalness .

A bounded linear operator $\mathrm{Z}$ is normal if it commutes with its adjoint : $\mathbf{Z} \mathbf{Z}=\mathbf{Z Z}^{*}$. The second step of the proof of Theorem 5.3 on equivalence of asymptotic stability and observability for normal operators is a simplification of observability condition

Theorem 5.1. If $\mathrm{Z}$ is a normal bounded linear operator then the observability condition (3.1) with $\mathrm{W}=\left(\mathrm{Z}^{*}+\mathrm{Z}\right)$ is equivalent to $\operatorname{Ker}\left(\mathrm{Z}^{*}+\mathrm{Z}\right)=\{0\}$.

Proof. If $Z$ is a normal linear bounded operator then

$$
\operatorname{Ker}\left(Z^{*}+Z\right) Z^{n}=\operatorname{Ker} Z^{n}\left(Z^{*}+Z\right) \geq \operatorname{Ker}\left(Z^{*}+Z\right) \text { for all } n \geq 0 \text {. }
$$

To show the reverse inclusion we argue as follows . Let $x \in \operatorname{Ker} Z\left(Z^{*}+Z\right)$. Then by normalness of $Z$ we obtain : $\left\|\left(Z^{*}+Z\right) x\right\|^{2}=\left(x \mid Z\left(Z^{*}+Z\right) x\right)+\left(Z\left(Z^{*}+Z\right) x \mid x\right)=0$. Thus $x \in \operatorname{Ker}\left(Z^{*}+Z\right)$. Hence $\operatorname{Ker}\left(Z^{*}+Z\right) \supseteq \operatorname{Ker} Z\left(Z^{*}+Z\right)=\operatorname{Ker}\left(Z^{*}+Z\right) Z^{n}$. This and (5.2) imply $\operatorname{Ker}\left(Z^{*}+Z\right) Z^{n}=\operatorname{Ker}\left(Z^{*}+Z\right)$ for all integers $n \geq 0$. Q.E.D.

The third step of the proof of Theorem 5.3 is asymptotic stability for selfadjoint operators. The proof is based on the theory of spectral measures, see [15] .

Lemma 5.2 . ON ASYMPTOTIC STABILITY FOR SELFADJOINT OPERATORS. If A is a weakly negative definite bounded selfadjoint linear operator in a Hilbert space $\mathrm{H}$ such that $\operatorname{Ker} \mathrm{A}=\{0\}$ then $\lim _{\mathrm{t} \rightarrow \infty}\left\|\mathrm{e}^{\mathrm{At}} \mathrm{x}\right\|=0$ for all $\mathrm{x} \in \mathrm{H}$.

Sketch of the proof.The spectral measure $\mu$ of the operator $A$ is projector-valued measure on the set of all Borel subsets of $[-\|A\|, 0)$ (since $A$ is weakly negative selfadjoint operator) such that

$$
\mathrm{e}^{\mathrm{At}} \mathrm{x}=\int_{[-\|\mathrm{A}\|, 0)} \mathrm{e}^{\zeta \mathrm{t}} \mu(\mathrm{d} \zeta) \mathrm{x}
$$

Regularity property of spectral measures implies that for every $\varepsilon>0$ there exists a natural number $\mathrm{N}(\mathrm{x}, \boldsymbol{\varepsilon})$ such that $\checkmark \int_{(-1 / n, 0)}(\mu(d \zeta) x, x)=\left\|\int_{(-1 / n, 0)} \mu(d \zeta) x\right\|=\left\|x-\int_{[-\|A\|,-1 / n]} \mu(d \zeta) x\right\| \leq \varepsilon$ whenever $n>N(x, \varepsilon)$.

Using this and (5.6) we approximate $u(t)$ with

$$
u_{n}(t)=e^{A t} \int_{[-\|A\|,-1 / n]} \mu(d \zeta) x=\int_{[-\|A\|,-1 / n]} e^{\zeta} \mu(d \zeta) x .
$$

Therefore $\lim _{t \rightarrow \infty}\left\|u_{n}(t)\right\|=0$. The proof follows easily from this . Q.E.D.

The following theorem generalizes the interesting result of R. K. Miller and A. N. Mitchell on evolution equations in Hilbert spaces with bounded normal infinitesimal generators .

Theorem 5.3 . ON EQUIVALENCE OF OBSERVABILITY AND ASYMPTOTIC STABILITY FOR NORMAL OPERATORS. Let $\mathrm{Z}$ be a normal bounded linear operator in a Hilbert space $\mathrm{H}$ such that $\mathrm{Z}^{*}+\mathrm{Z}$ 
is weakly negative definite. Then the pair $\left(\mathrm{Z}, \mathrm{Z}^{*}+\mathrm{Z}\right)$ is observable if and only if zero in $\mathrm{H}$ is a strongly asymptotically stable equilibrium point of (2.1).

Proof . Strong asymptotic stability implies observability by Theorem 3.3 and observability is equivalent to $\operatorname{Ker}\left(Z^{*}+Z\right)=\{0\}$ by Theorem 5.1. It remains to show that this condition implies asymptotic stability. So let $x \in B$ be arbitrary. By normality of $Z, e^{Z t}$ also commutes with its adjoint $\mathrm{e}^{Z^{*} t}$, see $[17]$, hence

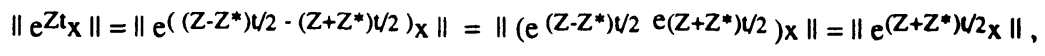

for $e^{\left(Z-Z^{*}\right) V_{2}}$ is a unitary operator since $\left(Z-Z^{*}\right) t / 2$ is skew-symmetric . But $\left(Z+Z^{*}\right) / 2$ is selfadjoint and its kernel is zero, hence by Lemma $5.2 \lim _{t \rightarrow \infty}\left\|e^{Z t_{x}}\right\|=\lim _{t \rightarrow \infty}\left\|e^{\left(Z+Z^{*}\right) V 2 x}\right\|=0$, which means asymptotic stability, for $x$ was arbitrary. Q.E.D.

Remark 5.4. R. Datko proved in [5] and [ 6] that in Hilbert space strict negative definiteness of $Z^{*}+Z$ implies an exponential decay of solutions to zero. Under the assumptions of Theorem 5.3 , where only weak negative definiteness is required, solutions may decay to zero much slower. This is illustrated below .

Example . Let us consider an equation of the form (2.1) in $H=L^{2}([0,1], C)$ with $\mathrm{Zu}(\mathrm{s})=-(\mathrm{K}+\beta \mathrm{s}) \mathrm{u}(\mathrm{s})$ where $\mathrm{K}$ is a nonnegative real number and $\mathrm{a}$ is complex with $\operatorname{Re}(\beta) \geq 0$. The solution may be easily found : $u(t, s)=u(0, s) e^{-(K+\beta s) t}$. It follows that $Z^{*} u(s)=-\left(K+\beta^{*} s\right) u(s)$ where $\beta^{*}$ is the number conjugate to $\beta$. The operator $Z^{*}+Z$ is strictly negative definite if $K>0$ for $\left(Z^{*}+Z\right) u(s)=-2(K+\operatorname{Re}(\beta)) u(s)$. In this case Datko's theory is applicable , hence norms of all solutions tend exponentially to zero. Indeed :

$$
\|\mathrm{u}(\mathrm{t}, .)\|=\mathrm{e}^{-\mathrm{Kt}} \sqrt{ }\left\{\int_{[0,1]} \mathrm{e}^{-2 \operatorname{Re}(\beta) s \psi} \mathrm{u}(0, \sigma) \mid 2 \mathrm{~d} \sigma\right\} \leq\|\mathrm{u}(0, .)\| \mathrm{e}^{-\mathrm{Kt}} \text {. }
$$

Let $K=0$. Then $Z^{*}+Z$ is weakly negative if $\operatorname{Re}(\beta)>0$, but it is not strictly negative definite, hence Datko's theory is not applicable, since zero belongs to the spectrum of $Z^{*}+Z$. However $Z^{*}$ and $Z$ commute. Therefore Theorem 5.3 implies strong asymptotic stability in this case. Let us take for example $\mathrm{u}(0, \mathrm{~s})=\mathrm{s}^{-\alpha}$, where $0<\alpha<1 / 2$, as initial data. Then the following is correct :

$$
\begin{aligned}
& \lim _{\mathrm{t} \rightarrow \infty} \mathrm{t}^{1-2 \alpha}\|\mathrm{u}(\mathrm{t}, .)\|^{2}=\lim _{\mathrm{t} \rightarrow \infty} \mathrm{t}^{1-2 \alpha} \int_{[0,1]} \mathrm{e}^{-2 \operatorname{Re}(\beta) s t} \mathrm{~s}^{-2 \alpha d s} \\
= & (\operatorname{Re}(\beta))^{2 \alpha-1} \lim _{\mathrm{t} \rightarrow \infty} \int_{[0, \operatorname{Re}(\beta) \mathrm{t}]} \mathrm{e}^{-2 \mathrm{~s}} \mathrm{~s}^{-2 \alpha} \mathrm{ds}=(\operatorname{Re}(\beta))^{2 \alpha-1} \int_{[0, \infty]} \mathrm{e}^{-2 s} \mathrm{~s}^{-2 \alpha} \mathrm{ds} .
\end{aligned}
$$

thus $\|u(t,)$.$\| tends to zero like t^{\alpha-1 / 2}$. Convergence may be arbitrarily slow if $\alpha$ is close to $1 / 2$. If $\operatorname{Re}(\alpha)=0$ then the above estimates break down and one obtains weak asymptotic stability as was shown the last example in the section 2 , despite that observability is lost .

\section{REFERENCES.}

[1] A. ARIS , G. R. Gevalas , On the theory of reactions in continuous mixtures . Phil. Trans. of Royal Soc. London, ser A, vol. 26(1966), pp. $351-393$.

[2] E. N. CHUKWU, Global behavior of retarded functional differential equations , part II . Paper in preparation .

[3] E.N. CHUKWU, A new invariance principle of functional differential equations of neutral type and applications. Paper in preparation .

[4] CODDINGTON, LEVINSON, Theory of linear differential equations, McGraw Hill , New York , 1955.

[5] R. DATKo , Extending a theorem of A. M. Lyapunov to Hilbert space . J. Math. Anal. Apl. , Vol. 32(1970) , pp. $610-616$

[6] R. DATKO , Extending a theorem of A. M. Lyapunov to semigroups of operators . 
J. Math. Anal. Apl. , Vol. 24(1968), pp. $290-295$.

171 N. DUNFORD, J.T. SCHWARTZ, Linear Operators, vol. 1 , International Publishers, New York, 1958.

[ 8 ] I. C. GOHBERG , M. G. KREIN , Theory and applications of Volterra operators in Hilbert space . AMS, Providence, 1970 .

[9] J.R. HADDOCK, J. TERJEKI, Lyapunov-Razumikhin functions and invariance principle for functional differential equations. Journ. of Diff. Eq. , 48(1983) , 95- 122 .

[ 10 ] J. K. HaLE, Dynamical systems and stability . J. Math. Anal. \& Apl. , Vol. 26(1969), pp. 39 - 59 .

[11] J. K. HALE , Ordinary differential equations, Wiley-Interscience, New York, 1969.

[12] A. HARAUX , Stabilization of trajectories for some weakly damped hyperbolic equations Journ. of Diff. Eq. , vol 59(1985) , 145-154 .

[13] J. P. LASALLE, An invariance principle in the theory of stability, Int. Symp. on Diff. Eq. and Dyn. Syst. , p. 277 , Academic Press, New York, 1967 , Editors : J. P. LaSalle , J. K. Hale .

[ 14 ] R. K. MILler , A.N. Mrtchell, Asymptotic stability of systems : Results involving system topology. SIAM J. Opt. Contr. , Vol. 18(1980), pp. $181-190$.

[14] W. MLAK, Introduction into the theory of Hilbert spaces, Warsaw , PWN , 1970.

[16 ] A. PAZY, Semigroups of linear operators and applications to partial differential equations. Springer-Verlag , Berlin , 1983.

[17] M. REED, B. SIMON , Methods of modern mathematical physics, Acad. Press , New York, 1980

[ 18 ] M. SLEMROD , Asymptotic stability of a class of abstract dynamical systems . J. Diff. Eq. , Vol. 7(1970), pp. 584 - 600 .

[19] R. TRIGGIANI , Controllability and observability in Banach spaces with bounded operators . SIAM J. Opt. Contr. , Vol. 13(1975), pp. 462 - 491.

[ 20 ] K. Yosida , Functional analysis, Springer-Verlag , Berlin 1978 .

[ 21 ] G. WEISS , Weak LP stability of a linear semigroup on a Hilbert space implies exponential stability . Journ. of Diff. Eq. , vol 76(1988) , 269-285 . 


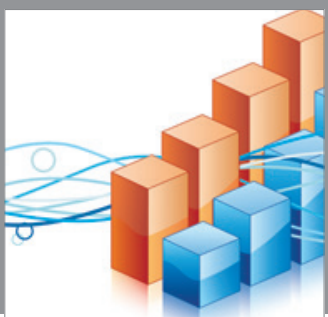

Advances in

Operations Research

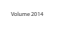

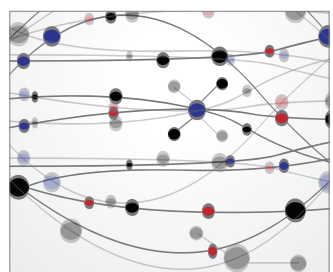

\section{The Scientific} World Journal
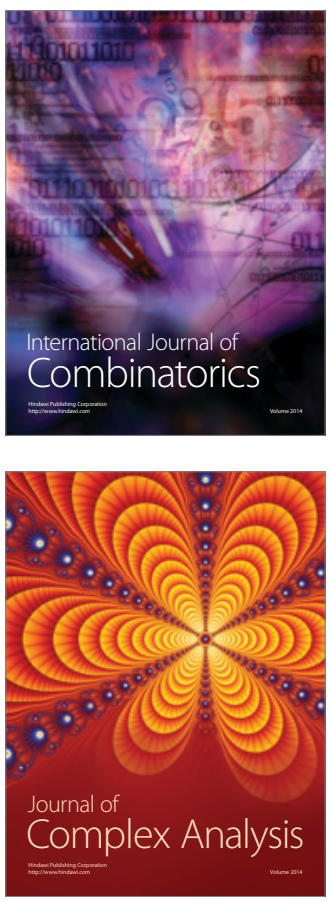

International Journal of

Mathematics and

Mathematical

Sciences
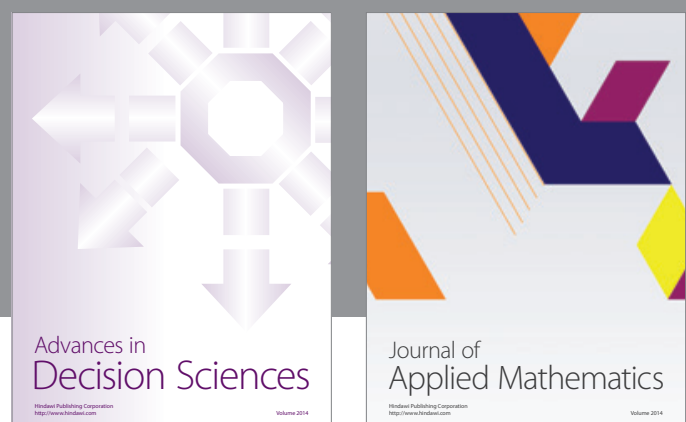

Journal of

Applied Mathematics
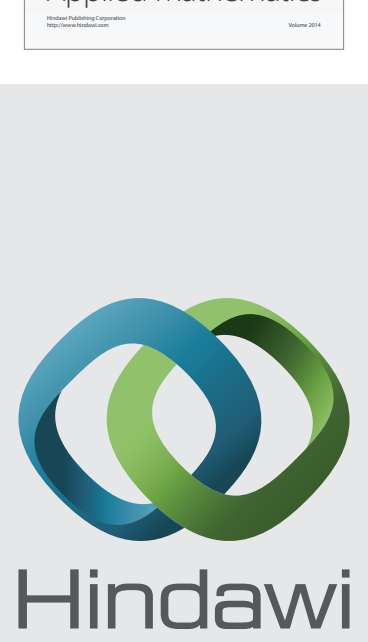

Submit your manuscripts at http://www.hindawi.com
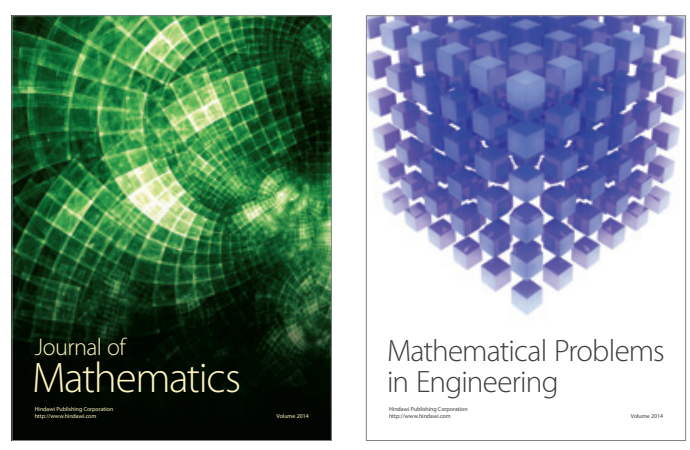

Mathematical Problems in Engineering
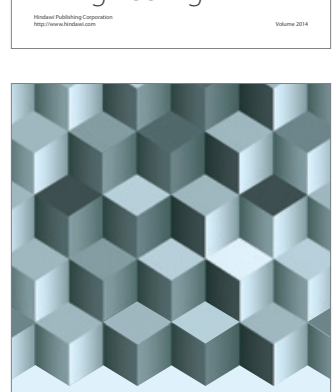

Journal of

Function Spaces
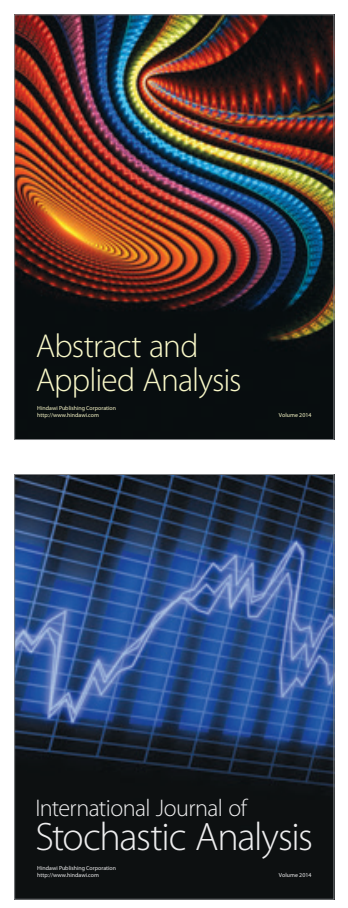

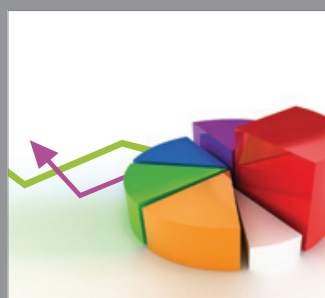

ournal of

Probability and Statistics

Promensencen
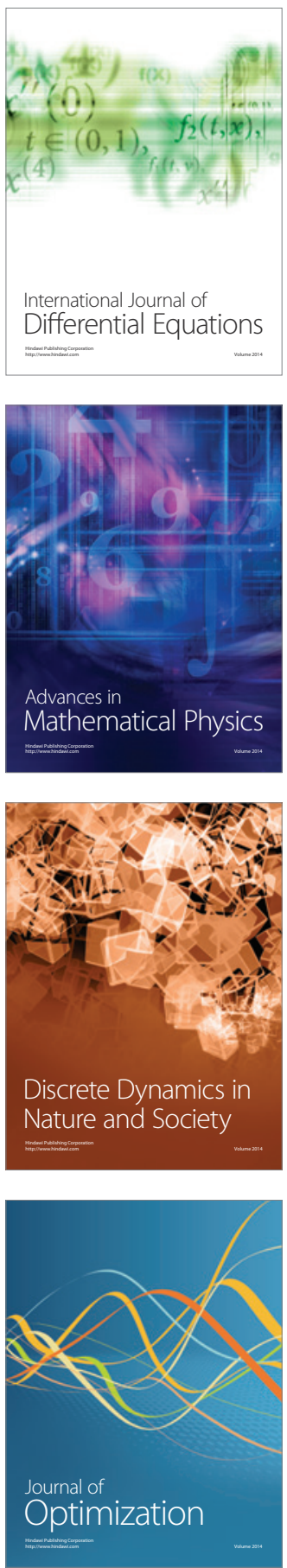